\title{
Four colour photometry of binary systems
}

\section{The early-type binary system CR Cassiopeiae ${ }^{\star, \star \star}$}

\author{
R. Clement and J. Fabregat \\ Departamento de Astronomía y Astrofísica, Universidad de Valencia, Dr. Moliner 50, E-46100 Burjassot, Valencia, Spain
}

Received February 17; accepted June 19, 1997

\begin{abstract}
This paper presents new uvby light curves of the detached eclipsing binary CR Cas together with some $\mathrm{H} \beta$ measurements. From the analysis of the photometry we have estimated preliminary values for interstellar reddening and distance, radiative parameters of both components and an approximation to the geometrical elements of the system. The intrinsic photometric indices are compatible with spectral types B0.5V + B1V.
\end{abstract}

Key words: stars: binaries: eclipsing — stars: earlytype - stars: fundamental parameters - stars: individual: CR Cas

\section{Introduction}

Mass is the fundamental parameter on which the evolution and the physical properties of a star at a given time depend directly. The mass of a star, its radius, effective temperature and abundances can only be accurately measured for double-lined eclipsing binaries with well determined light and radial velocity curves. For late-type stars these fundamental quantities are poorly defined. The current calibrations in the mass range $0.6<M / M_{\odot}<2.5$ are based on only a few stars. New and more accurate determinations of masses, radii, temperatures and abundances are thus needed for late-type stars. With this aim, we have undertaken a 6 year monitoring program of six binary systems classified in the literature as main sequence late-type stars and obtained good photometric uvby and $\mathrm{H} \beta$ light curves for three of them during 16 observing campaigns.

CR Cas is a detached binary system which was included in the photometric monitoring program of low mass

\footnotetext{
Send offprint requests to: $\mathrm{R}$. Clement

* Based on observations collected with the Spanish $1.5 \mathrm{~m}$. telescope at Calar Alto, Almería, Spain.

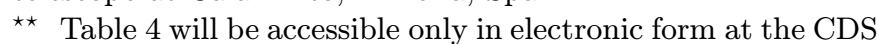
via anonymous ftp to cdsarc.u-strasbg.fr (130.79.128.5) or via http://cdsweb.u-strasbg.fr/Abstract.html
}

eclipsing binaries due to its classifications as spectral type G5 by Leung \& Scheneider (1977). The time of minimum is JD 2440526.279 and the period is 2.840147 days (Danielkiewicz-Krośniak \& Kurpińska-Winiarska 1994). Lacy (1992) observed CR Cas as a good candidate for determination of accurate absolute dimensions and obtained $V=11.370 \pm 0.015$ and $(B-V)=0.608 \pm 0.007$ from three photometric points.

In this paper we present the first uvby light curves for CR Cas. A few $\mathrm{H} \beta$ observations were also performed. Mean $\beta$ values for the comparison stars and binary system at different orbital phases are given. We also present the decoupling of the contribution of both components to the combined light, and their photometric analysis.

A preliminary analysis of the uvby photometry, with data from the two first campaigns, suggested that the system is a highly reddened early-type binary (Clement et al. 1993). The posterior careful analysis with the data presented in this paper gives the same result. Spectroscopic observations show the presence of He I lines, confirming this conclusion (Popper, private communication).

\section{The observations}

CR Cas was observed in three observational campaigns. The telescope used was the $1.5 \mathrm{~m}$ reflector of the Centro Astronómico Hispano-Alemán at the Calar Alto Observatory (Almería, Spain). The telescope was equipped either with a multipurpose one-channel photoelectric photometer (Lahulla \& Pensado 1981), or with a four-channel photometer (Florentin-Nielsen 1983), both using $u, v, b$, and $y$ filters as well as narrow and wide $\mathrm{H} \beta$ filters. The 377 points for the light curves, which cover the primary minimum at least three times and the secondary twice, were obtain in the following nights:

1. six nights in August 1991 using the four-channel photometer

2. one night in January 1992 using the four-channel photometer 
3. eleven nights in September 1995 using the one-channel photometer.

The instrumental system and transformation equations were computed separately for each individual run, following the procedure described in detail by Grønbech et al. (1976). In order to determine the atmospheric extinction, every night a set of 2 to 4 standard stars were observed more than four times at air masses ranging from 1 to 2 . In each observing run mean zero points were computed for the whole period, before determining the nightly extinction coefficients. Their mean values for the 18 nights considered, $0.182 \pm 0.047,0.057 \pm 0.010,0.045 \pm 0.006$ and $0.144 \pm 0.008$ for $y,(b-y), m_{1}$ and $c_{1}$ respectively, show the good behaviour of the atmosphere during those nights. We also determined the night corrections $L(n)$ within each observing period in order to obtain the magnitudes in the instrumental system. No significant variations between nights were found.

After extinction and night corrections, instrumental magnitudes were transformed to the Crawford \& Barnes (1970) and Crawford \& Mander (1966) standard systems. The standard stars were selected from the lists of Perry et al. (1987) and Olsen (private communication). A detailed description of the photometric reduction procedures, and an estimation of the accuracy of the photometry for the program campaigns can be found in Clement et al. (1997).

SAO 35044 and SAO 35197 were used as comparison and check star respectively for differential photometry. Its constancy was checked every night. Internal RMS errors of $0.009,0.005,0.014,0.023$, and 0.010 in $y,(b-y), m_{1}, c_{1}$ and $\beta$ for the 181 differences (SAO 35044 - SAO 35197) are of the same order as for the program objects and show no variability for the comparison stars during the period of observations. Due to the fact that the comparison stars are of different spectral type than CR Cas, they are not good matches for differential photometry, and some uncertainty due to extinction effects could be present in the differential light curves.

The differential magnitudes (CR Cas - SAO 35044) are listed in Table 4. Figures 1, 2, 3 and 4 show the differential light curves in each band. It is noticeable some level of variability at the bottom of the primary eclipse, mainly in the $u$ and $v$ bands. Moreover, the $\beta$ index is highly variable at all phases, with a dispersion at least three times higher than for the other stars measured in the same observing periods. This two facts led us to suggest that the secondary star could be an emission-line star (Clement et al. 1996), although no spectroscopic confirmation is available yet.

Photometric magnitudes and indices for the comparison stars and for CR Cas at eclipses and first quadrature are listed in Table 1.
Table 1. Standard photometry for CR Cas and the comparison stars

\begin{tabular}{|c|c|c|c|}
\hline & CR Cas & SAO 35044 & SAO 35197 \\
\hline$\alpha_{2000}$ & $23^{\mathrm{h}} 04^{\mathrm{m}} 51^{\mathrm{s}}$ & $23^{\mathrm{h}} 00^{\mathrm{m}} 15^{\mathrm{s}}$ & $23^{\mathrm{h}} 10^{\mathrm{m}} 43^{1}$ \\
\hline$\delta_{2000}$ & $59^{\circ} 34^{\prime} \quad 06^{\prime \prime}$ & $\begin{array}{lll}59^{\circ} & 27^{\prime} 05^{\prime \prime}\end{array}$ & $\begin{array}{lll}59^{\circ} & 00^{\prime} & 05^{\prime \prime}\end{array}$ \\
\hline Sp. type & $\mathrm{B} 0.5 \mathrm{~V}+\mathrm{B} 1 \mathrm{~V}$ & G8IV & G5 \\
\hline \multirow{2}{*}{$V(0.25)$} & 11.281 & 8.232 & 9.155 \\
\hline & 4 & 6 & 25 \\
\hline \multirow[t]{2}{*}{$V(0.00)$} & 12.182 & & \\
\hline & $\begin{array}{r}5 \\
710\end{array}$ & & \\
\hline$V(0.50)$ & 11.710 & & \\
\hline \multirow[t]{2}{*}{$(b-y)(0.25)$} & 0.503 & 0.646 & 0.583 \\
\hline & 4 & 1 & 6 \\
\hline \multirow[t]{2}{*}{$(b-y)(0.00)$} & 0.511 & & \\
\hline & 13 & & \\
\hline \multirow[t]{2}{*}{$(b-y)(0.50)$} & 0.495 & & \\
\hline & $\begin{array}{r}6 \\
-0104\end{array}$ & & \\
\hline$m_{1}(0.25)$ & $\begin{array}{r}-0.104 \\
10\end{array}$ & 0.345 & 0.276 \\
\hline$m_{1}(0.00)$ & $\begin{array}{r}-0.087 \\
32\end{array}$ & & \\
\hline$m_{1}(0.50)$ & $\begin{array}{r}-0.122 \\
11\end{array}$ & & \\
\hline \multirow[t]{2}{*}{$c_{1}(0.25)$} & 0.114 & 0.389 & 0.326 \\
\hline & 9 & 7 & 11 \\
\hline$c_{1}(0.00)$ & $\begin{array}{r}0.272 \\
34\end{array}$ & & \\
\hline \multirow[t]{2}{*}{$c_{1}(0.50)$} & 0.092 & & \\
\hline & 24 & & \\
\hline$\beta(0.25)$ & $\begin{array}{r}2.606 \\
6\end{array}$ & $\begin{array}{r}2.573 \\
2\end{array}$ & $\begin{array}{r}2.554 \\
13\end{array}$ \\
\hline \multirow[t]{2}{*}{$\beta(0.00)$} & 2.607 & & \\
\hline & 16 & & \\
\hline \multirow[t]{2}{*}{$\beta(0.50)$} & 2.602 & & \\
\hline & 1 & & \\
\hline
\end{tabular}

Table 2. Intrinsic magnitudes and indices

\begin{tabular}{lrrrrr}
\hline name & $y_{0}$ & $(b-y)_{0}$ & $m_{0}$ & $c_{0}$ & $\beta$ \\
\hline CR Cas Hot & 9.040 & -0.126 & 0.078 & -0.034 & 2.602 \\
CR Cas Cold & 2 & 6 & 11 & 24 & 1 \\
& 9.827 & -0.104 & 0.136 & 0.048 & 2.614 \\
& 13 & 22 & 52 & 84 & 9 \\
\hline
\end{tabular}

\section{Discussion}

\subsection{Interstellar reddening and spectral types}

We have calculated the magnitudes for the hot and cold components, assuming total secondary eclipse, that is, at the secondary minimum the only light contribution is from the bigger, hotter star. This last hypothesis is consistent with both components being main sequence stars, as we show below. The alternative hypothesis of a cooler, evolved star occulting its companion in the primary eclipse is less compatible with the value of the $\beta$ index at phase 0.0. Interstellar reddening for each component has been computed following the method described by Crawford (1978), and using the standard relations for the intrinsic indices given by Perry et al. (1987). We found 


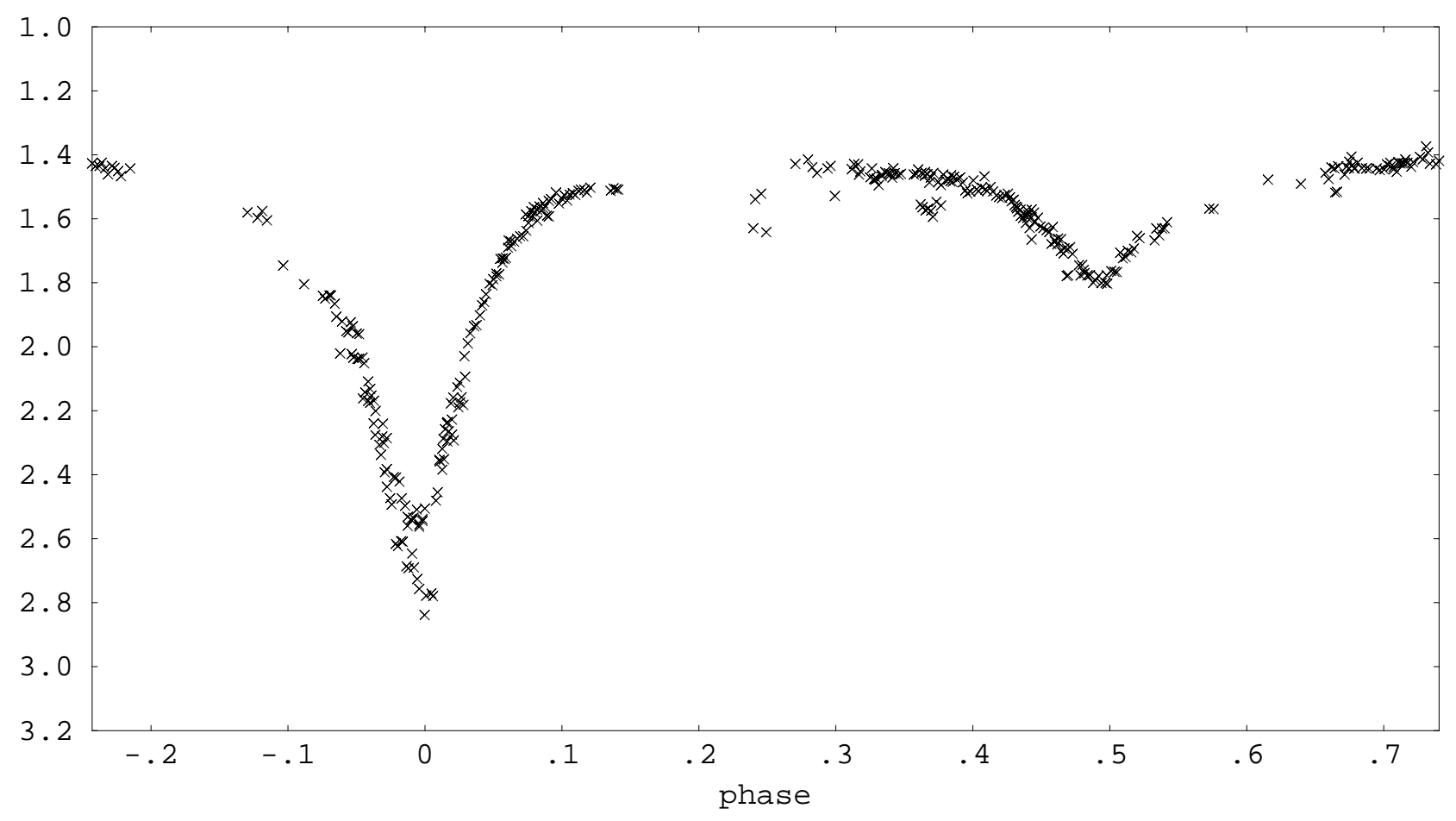

Fig. 1. CR Cas differential light curve. $u$ filter

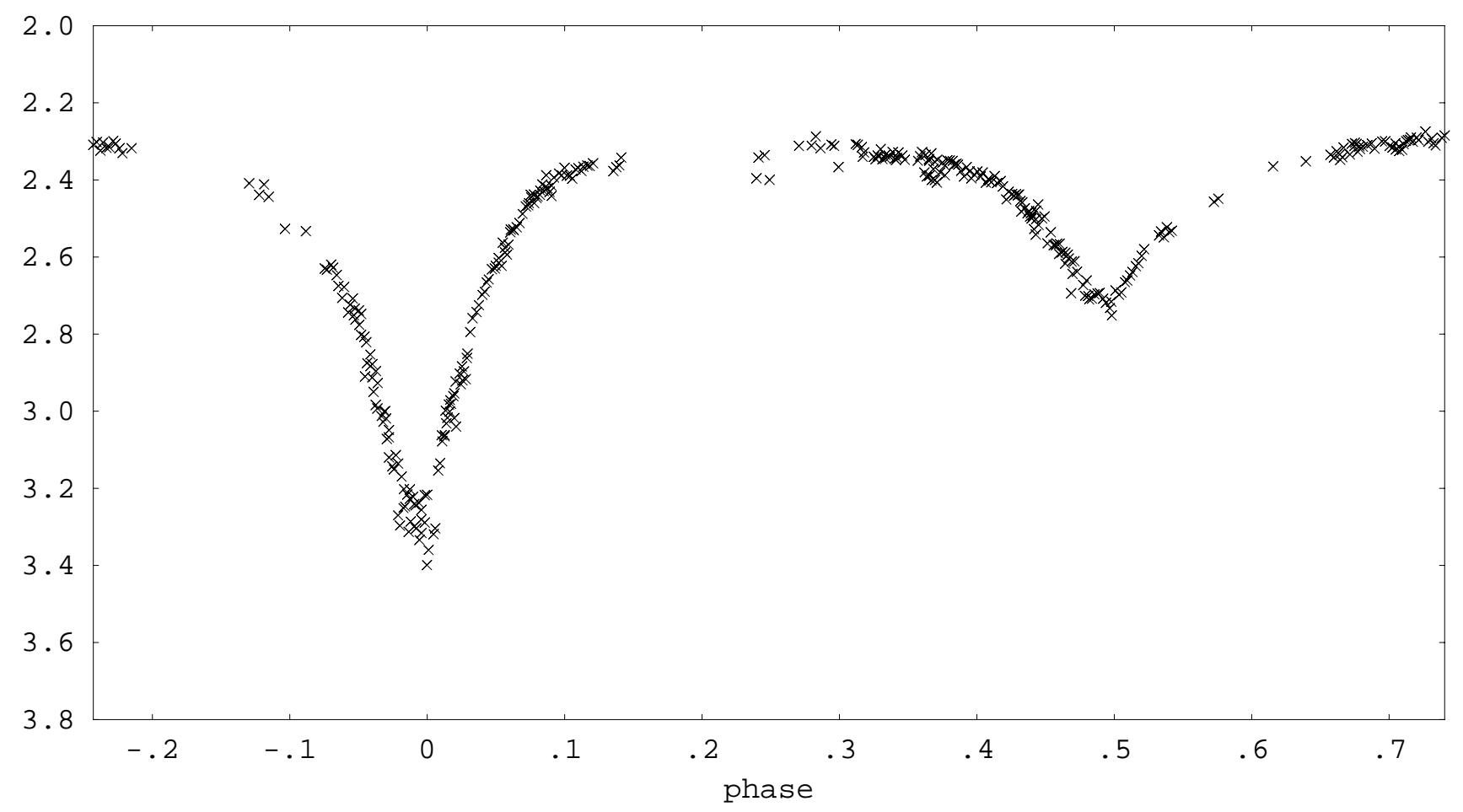

Fig. 2. CR Cas differential light curve. $v$ filter 


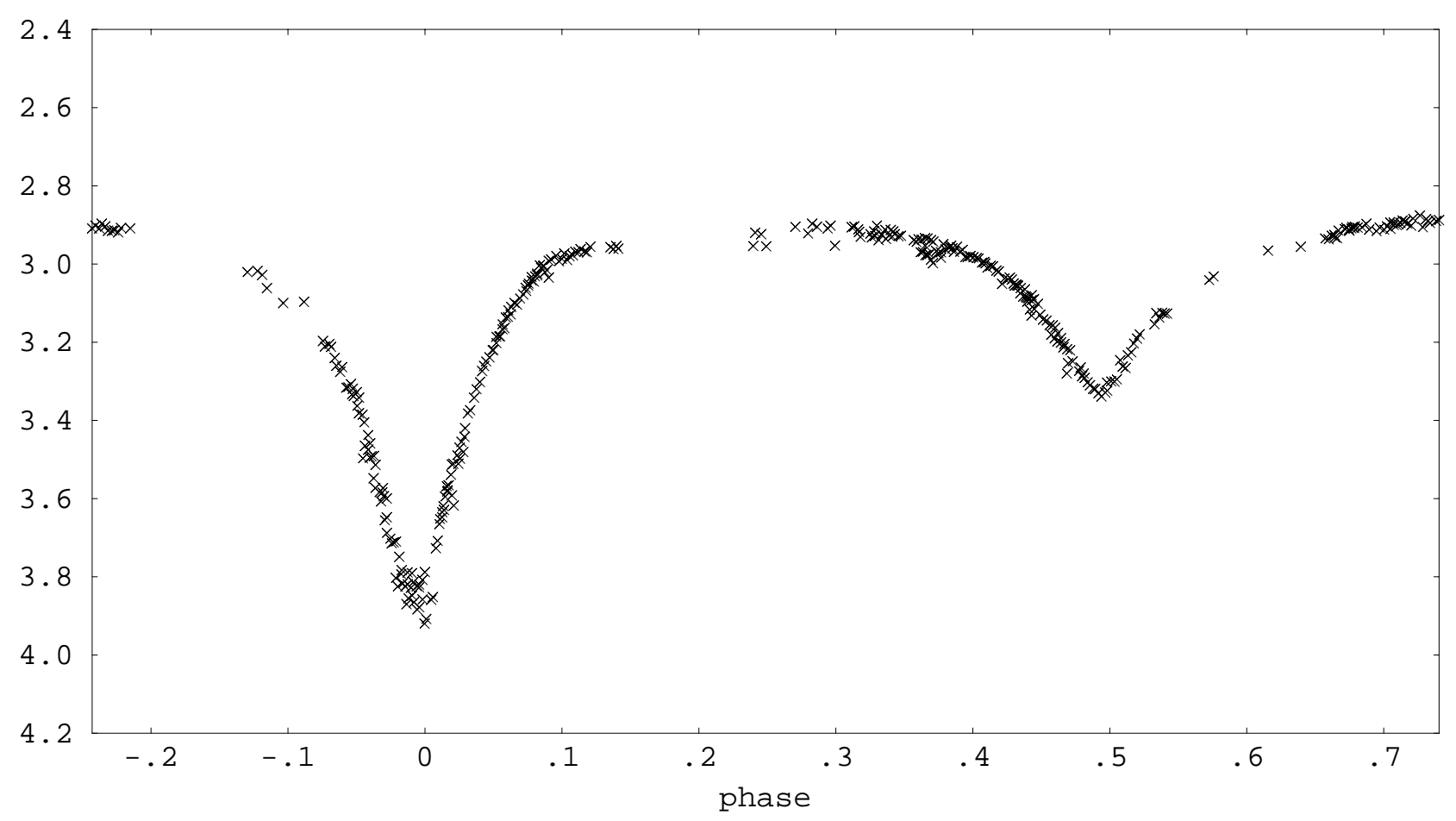

Fig. 3. CR Cas differential light curve. $b$ filter

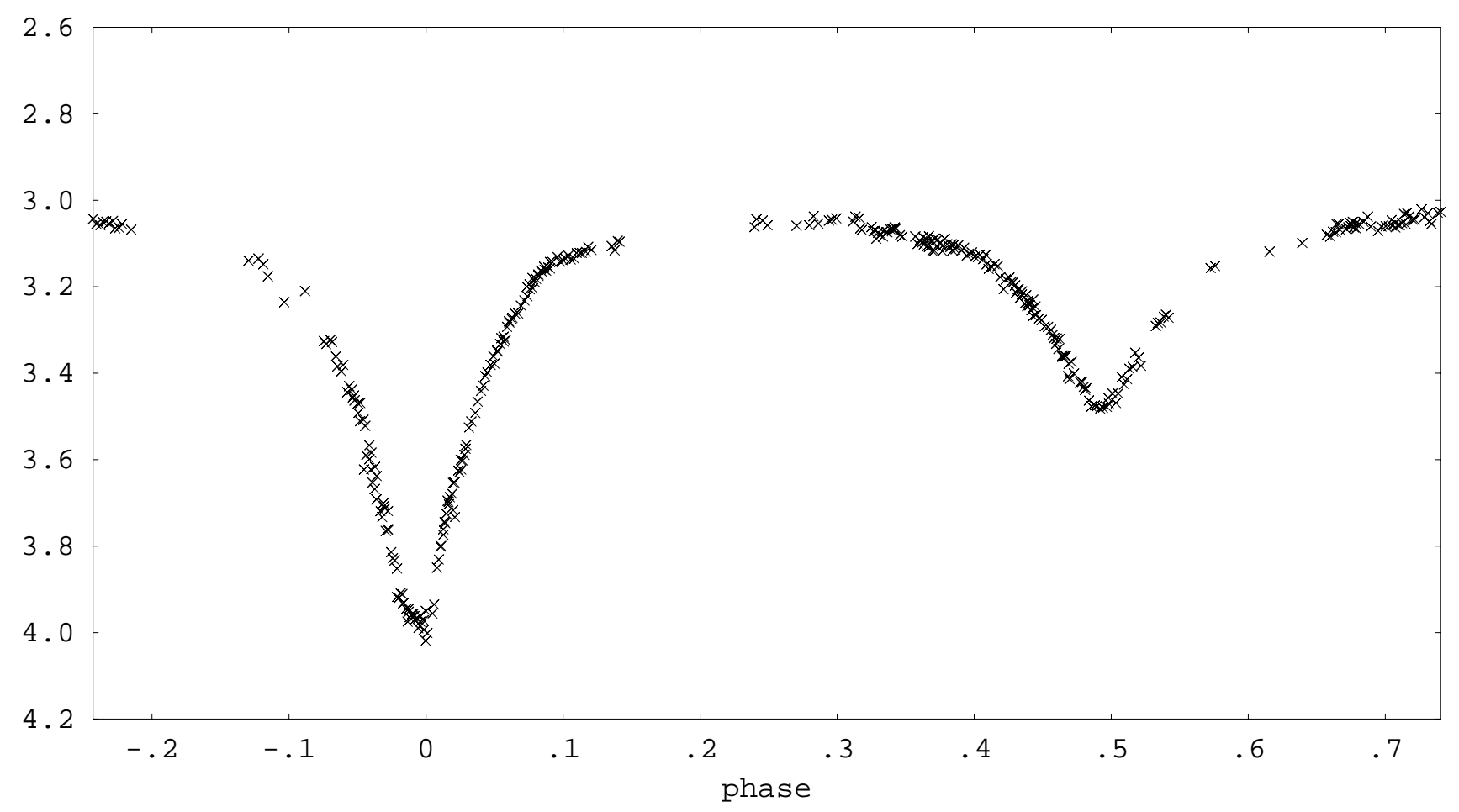

Fig. 4. CR Cas differential light curve. $y$ filter 
Table 3. Radiative Parameters for CR Cas

\begin{tabular}{lrrrrrrrrl}
\hline & $M_{V}$ & $F_{V}$ & $R / R_{\odot}$ & $T_{\text {eff }}(\mathrm{K})$ & $M_{\text {bol }}$ & $\log \left(L / L_{\odot}\right)$ & $M / M_{\odot}$ & $\mathrm{d}(\mathrm{pc})$ & $\mathrm{MK}$ \\
\hline CR Cas Hot & -3.8 & 4.19 & 6.7 & 26010 & -6.4 & 4.26 & 12.6 & 3680 & $\mathrm{~B} 0.5 \mathrm{~V}$ \\
& 4 & 3 & 1.6 & 400 & 5 & 20 & 1.0 & 750 & \\
CR Cas Cold & -3.0 & 4.14 & 5.8 & 22780 & -5.3 & 3.91 & 9.8 & 3680 & $\mathrm{~B} 1 \mathrm{~V}$ \\
& 4 & 3 & 1.4 & 530 & 5 & 21 & 1.0 & 750 & \\
\hline
\end{tabular}

$E(b-y)=0.617$ and $E(b-y)=0.638$ for the primary and secondary stars respectively. We adopted the mean of the two determinations, weighted with the rms dispersion of the $c_{1}$ index, which gives a value of $E(b-y)=0.621$ as the reddening for the system. Table 2 list the intrinsic magnitudes and indices for both components. Errors given for the primary star are the rms dispersion of the six points averaged at the bottom of the secondary eclipse. For the cold component the errors listed are the propagation errors through the formulae used in the decoupling. The intrinsic indices are compatible with spectral types $\mathrm{B} 0.5 \mathrm{~V}$ and $\mathrm{B} 1 \mathrm{~V}$, assuming the mean values as a function of spectral type given by Crawford (1978).

\subsection{Radiative parameters}

We used the empirical calibration of Balona \& Shobbrook (1984) to calculate the absolute magnitudes and distances of both components separately. The obtained distances, $3550 \pm 700$ and $3800 \pm 750$ parsec, show a good internal agreement and are coherent with the reddening estimated. We will assume the weighted mean, $3650 \pm 750$ as the distance of the system. Final absolute magnitudes have been calculated using the observed apparent magnitudes and the assumed mean distance.

To estimate the radii we have first computed the visual surface brightness parameter $F_{V}$ (Barnes \& Evans 1976), by means of the calibration in terms of $(b-y)$ given by Moon (1984). The effective temperature has been derived comparing the obtained photometric indices with the synthetic indices computed by Lester et al. (1986), using the interpolating formula given by Balona (1994). From the obtained temperatures we have derived the bolometric corrections using the Balona (1994) relationship. Masses have been estimated from the evolutionary models of Claret \& Giménez (1992), using the interpolating formula computed by Balona (1994). The computed values are listed in Table 3.

\subsection{Geometrical parameters}

Although there are not radial velocity curves published for this binary system, we have done a preliminary estimation of the radii of both components relative to the orbit $\left(r_{\mathrm{p}}\right.$, $r_{\mathrm{s}}$ ) and the inclination of the orbit $(i)$, by using the EBOP code, written by Etzel (1975). Initial values to run EBOP were assumed from Table 3. Because the light curves show appreciable variation at the bottom of the primary eclipse, specially in the $u$ filter (see Figs. 1, 2, 3 and 4), we computed the EBOP solution with the data obtained in the last campaign (292 points). We found that the best theoretical light curves correspond to stars with $r_{\mathrm{p}}=0.35$ and $r_{\mathrm{s}}=0.29$ for the hot and cold component respectively, and $i=85^{\circ}$.

\section{Conclusions}

Our photometric study shows that CR Cas is a detached eclipsing binary whose components are early B type, main sequence stars. In the recent compilation of Andersen (1991), it is shown that only three eclipsing binaries with components earlier than B2 have their astrophysical parameters determined with an accuracy better than $2 \%$. In three spectra of CR Cas obtained by Popper (1996) no separate lines from both components appear to be measurable, but he states that with this small number of spectra no firm conclusion can be reached. Further spectroscopic work would be of great value in order to discern whether CR Cas is a double-lined eclipsing binary. If this is the case, CR Cas would be a good candidate for the accurate determination of the stellar astrophysical parameters in a range of masses in which the available systems are very scarce.

Acknowledgements. The $1.5 \mathrm{~m}$ telescope at Calar Alto is operated by the Observatorio Astronomico Nacional. We want to express our gratitude to the observatory staff. This work has been supported by the Spanish Comisión Interministerial de Ciencia y Tecnología (PB90-0001-C02).

\section{References}

Andersen J., 1991, A\&AR 3, 91

Balona L.A., 1994, MNRAS 268, 119

Balona L.A., Shobbrook R.R., 1984, MNRAS 211, 375

Barnes T.G., Evans D.S., 1976, MNRAS 174, 489

Claret A., Giménez A., 1992, A\&AS 96, 255

Clement R., Reglero V., García M., et al., 1993, in ASP Conf. Ser. 40, Inside the Stars, Weiss W.W., Baglin A. (eds.) p. 386

Clement R., Reglero V., Fabregat J., 1996, Be Star News. 31, 13

Clement R., García M., Reglero V., et al., 1997, A\&AS 123, 1

Crawford D.L., 1978, AJ 83, 48 
Crawford D.L., Mander J., 1966, AJ 71, 114

Crawford D.L., Barnes J.V., 1970, AJ 75, 978

Danielkiewicz-Krośniak E., Kurpińska-Winiarska M., 1994, International Supplement, Annuario Cracoviense, p. 65

Etzel P.B., 1975, Masters Thesis. San Diego State University Florentin-Nielsen R., 1983, Rep. Inst. Theor. Astrophys. Oslo 59,141

Grønbech B., Olsen E.H., Strömgren B., 1976, A\&AS 26, 155

Lacy C.H., 1977, ApJ 213, 458

\section{Lacy C.H., 1992, AJ 104, 801}

Lahulla J.F., Pensado J., 1981, Bol. Obs. Astron. de Madrid Vol. XI, 1

Lester J.B., Gray R.O., Kurucz R.L., 1986, ApJS 61, 509

Leung K.C., Schneider D.P., 1977, AJ 211, 844

Moon T.T., 1984, MNRAS 211, 21P

Perry, C.L., Olsen, E.H., Crawford, D.L., 1987, PASP 99, 1184

Popper D.M., 1996, ApJS 106, 133 